acidité de $0 \mathrm{gr}$. 04 exprimée en acide lactique et 100 gr. de caséine une acidité de 2 gr., soit $2 \%$.

On peut aussi employer une burette queleonque graduée en $1 / 10$ de cc. et de la soude $\mathrm{N} / 10$; dans ce cas en prenant les $9 / 10$ du nombre trouvé on aura l'acidité exprimée en acide lactique.

Avec ce procédé d'analyse on ajoute l'acidité propre de la caséine à l'acidité due à la présence de l'acide lactique, mais dans la pratique industrielle cela ne parait pas avoir d'importance. Par contre on obtient les mêmes nombres, avec la même easéine, quelle que soit la grosseur du grain. Il y a seulement intérêt à broyer finement les caséines, de façon ì opérer plus rapidement.

La caséine à la présure a, en employant cette méthode, une acidité variant de 1,5 à $3 \%$ suivant que la caillebotte a été plus ou moins bien lavée ; l'acidité de la caséine lactique est le plus souvent comprise entre 6 et $8 \%$.

\title{
LA POUDRE DE LAIT ET L'INDUSTRIE FROMAGËRE
}

\author{
par M. Ch. PORCHER,
}

L'idée n'est pas neuve de faire des fromages avee de la poudre de lait, on peut dire qu'elle est née en même temps que l'industrie du lait desséché.

Bien des essais ont été faits autrefois pour faire des fromages avec du lait sec. Ils ont été plus ou moins heureux, mais il faut reconnaître que l'emploi du chlorure de calcium, préconisé par L. LINDET, lequel donne déjà de si bons résultats dans l'industrie fromagère, ne peutqu'encourager à recourir à la poudre de lait dans la fabrication des fromages.

Dans la $2^{\mathrm{e}}$ édition de mon livre sur le lait desséché (chapitre XIV, pp. 187-190), j'a consacré un court chapitre à la question. Je pense qu'il est intéressant que je le donne ici en son entier, ear il servira d'introduction à l'article qui suit sur un essai de fabrication du fromage avec du lait sec, que j'ai tenté l'an passé, avec le concours de M. DesRantes, de la Société Laitière Moderne de Lyon.

$$
\text { *** }
$$

Si l'on a pensé à faire du lait reconstitué au moyen de la poudre maigre et du beurre, c'était, d'une part, pour répondre aux moments de disette passagère possible, aux accidents provoqués par des chaleurs excessives, quand l'approvisionnement normal en lait frais est troublé et diminué ; c'était, d'autre part, pour sţabiliser le marché des produits de la laiterie, résoudre le problème de l'utilisation d'une surabondance de lait à certaines époques de l'année, et somme toute, savoir utiliser au mieux une marchandise périsable entre toutes et dont la production est malheureusement irrégulière. 
On peut penser à employer la poudre obtenue l'été, bien conditionnée et conservée, pour fabriquer, l'hiver, du fromage. Le fromage est un produit qui se vend relativement cher, qui permet de tirer de la matière première : le lait, un prix rémunérateur ; mais, son industrie est souvent compromise pendant les temps chauds; la fabrication en est irréguière et les produits se vendent mal, surtout quand il s'agit de fromages à pâte molle.

II peut naturellement venir à l'esprit d'utiliser le lait reconstitué dans le sens de lait refait pour faire du fromage, car, à notre avis, ce serait une erreur que de s'adresser à la dissolution de la poudre grasse préparée l'été pour servir, en hiver, de matière première à la fabrication du fromage.

Une pareille solution appelle des objections auxquelles il peut être aisément répondu.

Nous savons que des tentatives; sur une petite échelle d'ailleurs, ont été faites pour fabriquer des fromages avee de la poudre de lait; elles sont loin d'avoir donné des résultats décourageants, et l'on peut estimer qu'il y a là, pour l'avenir, un débouché important pour l'industrie de la poudre. Mais, ainsi que nous l'avons vu dans les pages qui précèdent, il y a poudre et poudre, et celle à laquelle on peut penser dans le but d'en faire du fromage doit répondre à certaines exigences compatibles avee la bonne marche de l'emprésurage. Les poudres à utiliser doivent done bien se dissoudre; les propriétés de leur colloïdes doivent être celles $\mathrm{du}$ lait originel, ou du moins n'avoir subi du fait de la dessiccation qu'une altération minima qui ne puisse gêner en rien la coagulation par la présure: L'hydrophilie de ces colloïdes, la conservation de l'équilibre qui s'est établi entre eux, colloïdes organiques et colloïdes salins, ne doivent avoir été touchées qu'au minimum. Au cas où l'atteinte serait plus prononcée, sans l'être trop, nous avons heureusement un moyen excellent pour parer à ces inconvénients, c'est l'emploi du chlorure de calcium. Déjà pour le lait liquide courant, les recherches de L. LINDET qui ont été rappelées plus haut, ont montré les résultats heureux que donnait dans l'industrie de la fromagerie, l'emploi régulier du chlorure de calcium ajouté au lait avant l'emprésurage; le caillage est -plus rapide, les pâtes sont plus fermes et s'égouttent mieux.

Si nous considérons maintenant la fabrication des fromages du côté microbien, nous savons que nombreuses ont été les tentatives faites pour n'utiliser que des laits pasteurisés, puis réensemencé dans des conditions bien déterminées avec des levains lactiques, dont on soit maître tant du côté de la qualité des microbes qui s'y trouvent, que du côté de l'acidité de ce levain. Or, ee que la pasteurisation réalise avec le laît liquide courant est obtenu ipso faclo dans la fabrication des poudres de lait; celles-ci, nous l'avons vu, sont peu microbiennes, elles ont été débarrassées par la pasteurisation préalable, ensuite par la dessiccation 
des bactéries pathogènes et de la presque totalité de leur flore banale. Lo lait rezonstitué avec ces poudres a toutes les propriétés du lait pasteurisé, à haute température s'il s'agit de poudre "sur cylindres ", du lait ayant été soumis à la pasteurisation basse, s’il s'agit de poudre " par brouillard ", surtout de la poudre obtenue par centrifugation.

$$
*^{*} *
$$

Des lignes qui précèdent, lesquelles résument en l'adaptant à la question visée dans ce chapitre bien des acquisitions relevées dans les pages antérieures, il est farile de conclure qu'entre les poudres dont il est question dans ce livre, il est un choix à faire en vue de leur utilisation dans ''industrie fromagère. C'est, à n'en pas douter, aux poudres obtenues " par brouillard ", notamment à celles qui ont recours à la centrifugation, qu'il faudra s'adresser. Chez elles, l'action de la chaleur n'a jamais dépassé celle qui est mise en œuvre dans la pasteurisation basse. Les propriétés biochimiques sont peu touchées, l'équilibre colloïdal n'est que légerement atteint, la solubilité est excellente, elles donnent la réaction de la peroxydase; le lait reconstitué avec elles caille très bien par la présure, en donnant un caillot ferme, peut-être un peu plus tardivement que le lait originel, ce à quoi it est facile de remédier en ajoutant à la solution une petite quantité de chlorure de calcium.

Le lait reconstitué devra être ensemencé d'une quantité déterminée d'un levain lastique. Bien mieux qu'aves le lait ordinaire, on pourra être maître de la marche de l'acidification du lait, laquelle joue un rôle si dominant dans l'emprésurage.

En résumé, la poudre de lait est appelée à donner des résultats inté ressants dans l'industrie fromagère. Au début, il y aura des tâtonnements dont on ne devra pas s'étonner, puisqu'on travaillera sur une matière nouvelle, mais aujourd'hui que l'on connaît parfaitement toutes les conditions de l'emprésurage : acidité, quantité de présure, durée de la coagulation, on aura vite fait de résoudre les premières difficultés rencontrées.

La poudre de lait pourra-t-elle servir indistinctement à fabriquer tout les fromages : pâtes molles et pâtes fermes, fromage à moisissures et fromages euits, ete. C'est là une question à laquelle répondra la pratique.

Nous avons tenu simplement dans ces lignes à marquer l'importance considérable d'un débouché pour l'industrie de la poudre de lait, à souligner, théoriquement du moins, car, à ma connaissance, les essajs ne sont pas encore assez nombreux pour en tirer une opinion ferme, la possibinité de partir de la poudre pour fabriquer des fromages.

Nous savons que la question est agitée dans les milieux qui souffrent justement de la surabondance de lait à certains moments de l'année, et qui envisageraient l'utilisation des excédents sous une forme rémunératrice. Ce n'est pas d'aujourd'hui qu'ils avaient pensé à fabriquer de 
la poudre, mais c'était pour la boulangerie, la chocolaterie, etc. ; ici, le rendement financier, quoique bon, est cependant assez aléatoire vu la concurrence. S'il devient possible de faire du fromage, la chose apparaît autrement intéressante, car la consommation du fromage va en augmentant, et la demande, l'hiver, est toujours importante et les prix sont soutenus. Mais faisons hautement remarquer aux industriels dont l'intention serait de faire du fromage avec de la poudre de lait, qu'ils aient à orienter leur fabrication de lait desséché dans une direction donnée, qu'iis se gardent bien de dessécher des laits acides, et surtout de les neutraliser au préalable; on obtiendrait des poudres alcalines dont l'emprésurage pourrait conduire à des résultats défectueux. même après l'addition du levain lactique.

La fabrication des fromages en partant de la poudre maigre doit faire appel à une addition de matière grasse, car nous ne songeons nullement ici aux fromages maigres; ce sont des produits meilleurs que nous envisageons.

Sous quelle forme alors la matière grasse sera-t-elle ajoutée au lait maigre reconstitué? Sera-ce du beurre fait de crème douce qu'on émulsionnera eomme il a été vu plus haut? Ou mélangera-t-on en proportions convenables au lait écrémé reconstitué du lait entier frais ou de la crème? Il y a plusieurs modalités à envisager et sur lesquelles la pratique sera à même de nous fixer. 11 importe cependant de relever que si l'on avait recours à du lait entier ou à de la crème, il serait indiqué de les pasteuriser au préalable avant de les ajouter au lait maigre reconstitué, sinon on associerait deux états bactériens différents, celui de la poudre maigre, lequel se traduit par une relative asepsie, et celui du lait entier ou de la crème fraîche, plutôt mal connu.

Nous conclurons en disant que nous n'avons entendu fournic ici que des directions générales, souligner les principales objections, et donner, nous le pensons du moins, le moyen d'y répondre; mais nous nous tromperions fort si l'emploi des bonnes poudres de lait, bonnes au triple point de vue: microbien, physico-chimique et colloïdal, ne prenait pas place un jour dans l'industrie fromagère.

\section{UN ESSAI DE FABRICATION DE FROIMAGE AVEC DU LAIT SEC}

par MM. Ch. PORCHER et R. DESRANTES.

Il importe tout d'abord de donner en quelques mots l'histoire de la poudre qui a servi à faire les essais que nous avons tentés, M. DEsRantes et moi l'an dernier.

Il s'agissait d'une poudre maigre fabriquée par le procédé KRAUSE et qui m'avait été adressée par la firme italienne PoLANGHI de Codogno (Lombardie); c'était une poudre fort soluble dans l'eau, très appétissante. 